\title{
SMOOTH FUNCTIONS WITH UNCOUNTABLY MANY ZEROS
}

\author{
J. A. CONEJERO, G. A. MUÑOZ-FERnÁNDEZ, M. MURILlo ARCILA, \\ AND J.B. SEOANE-SEPÚLVEDA
}

\begin{abstract}
In this short note we show that there exist uncountably generated algebras every non-zero element of which is a smooth function having uncountably many zeros. This result complements some recent ones by Enflo et al. $[7,9]$.
\end{abstract}

As it nowadays is common terminology, a subset $M$ of a topological vector space $X$ is called lineable (respectively, spaceable) in $X$ if there exists an infinite dimensional linear space (respectively, infinite dimensional closed linear space) $Y \subset M \cup\{0\}$. Recently there have been several results regarding the linear structure of certain subsets of real functions having a large set of zeros. For instance, in [9], Enflo et al. proved that, for every infinite dimensional closed subspace $X$ of $\mathcal{C}[0,1]$, the set of functions in $X$ having infinitely many zeros in $[0,1]$ is spaceable in $X$. Also, in [7], Conejero et al. constructed an algebra of functions $\mathcal{A}$ enjoying the following properties: (i) $\mathcal{A}$ is uncountably infinitely generated (that is, the cardinality of a minimal system of generators of $\mathcal{A}$ is uncountable), (ii) every nonzero element of $\mathcal{A}$ is nowhere analytic, (iii) $\mathcal{A} \subset \mathcal{C}^{\infty}(\mathbb{R})$, (iv) every element of $\mathcal{A}$ has infinitely many zeros in $\mathbb{R}$, and (v) for every $f \in \mathcal{A} \backslash\{0\}$ and $n \in \mathbb{N}, f^{(n)}$ (the $n$-th derivative of $f$ ) enjoys the same properties as the elements in $\mathcal{A} \backslash\{0\}$. Also, let us recall the notion of algebrability (see, e.g. $[1-5,10]$ ). Given an algebra $\mathcal{A}$, a subset $\mathcal{B} \subset \mathcal{A}$, and a cardinal number $\kappa$, we say that $\mathcal{B}$ is: (i) algebrable if there is a subalgebra $\mathcal{C}$ of $\mathcal{A}$ so that $\mathcal{C} \subset \mathcal{B} \cup\{0\}$ and the cardinality of any system of generators of $\mathcal{C}$ is infinite. (ii) $\kappa$-algebrable if there exists a $\kappa$-generated subalgebra $\mathcal{C}$ of $\mathcal{A}$ with $\mathcal{C} \subset \mathcal{B} \cup\{0\}$. (iii) strongly $\kappa$-algebrable if there exists a $\kappa$-generated free algebra $\mathcal{C}$ contained in $\mathcal{B} \cup\{0\}$.

On a totally different framework, and somehow related to the study of the set of zeros of functions on a given interval, Aron and Gurariy in 2003, asked whether there exists an infinite dimensional subspace of $\ell_{\infty}$ every non-zero element of which has a finite number of zeros. This question was recently answered, in the negative, in $[6]$.

Let us also recall that both of the results from $[7,9]$ share a common ground: The cardinality of the considered set of zeros was always countable. Of course, by means of a Baire category argument (as seen in [8]) one can show that almost every continuous function having zeros has, actually, an uncountable amount of them.

2010 Mathematics Subject Classification. 15A03, 26 B05.

Key words and phrases. lineability, spaceability, algebrability, zero set.

The first author was supported by Programa de Investigación y Desarrollo de la UPV (ref. SP20120700) and by MTM2013-47093-P. The third author was supported by MTM2013-47093-P and by a grant from the FPU program of MEC. The second and fourth authors were supported by MTM2012-34341. 


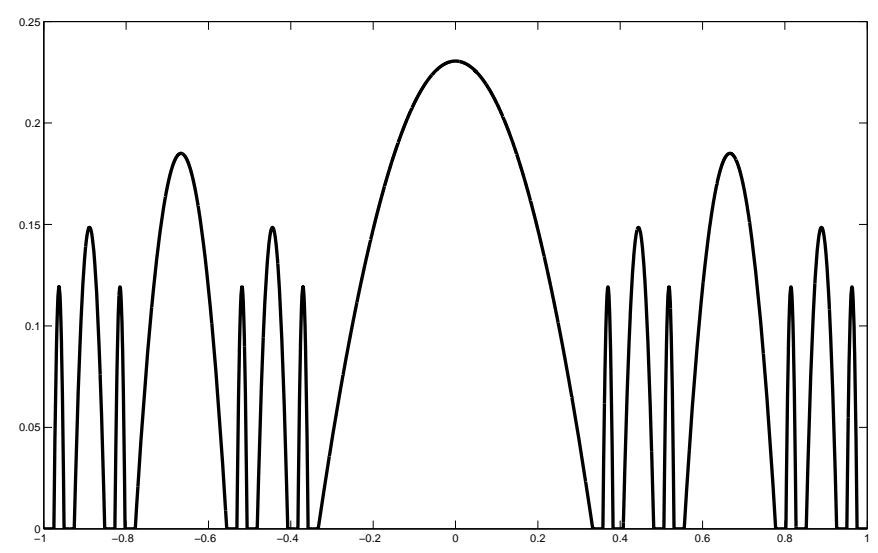

FiguRE 1. Sketch of $d(x)$ on $[-1,1]$.

In this short note we complement the previously mentioned results by proving, constructively, the following:

Theorem. The subset of smooth functions in $\mathbb{R}$ having a uncountable set of zeros is strongly $\mathfrak{c}$-algebrable.

Let us start by fixing $Z \subset \mathbb{R}$ with $|Z|=\mathfrak{c}$ and a function $0 \neq f \in \mathcal{C}^{\infty}(\mathbb{R})$ such that $f(z)=0$ for every $z \in Z$ and $f$ does not have horizontal asymptotes. Such a function can be defined as follows. Let $\mathfrak{C}$ be a copy of the Cantor set in the interval $[-1,1]$. Observe that $[-1,1] \backslash \mathfrak{C}=\bigcup_{n} I_{n}$, where the $I_{n}$ 's are pairwise disjoint open intervals. Now define the function $d:[-1,1] \rightarrow \mathbb{R}$ as

$$
d(x)= \begin{cases}k_{a_{n}, b_{n}} \cdot\left(x-a_{n}\right)\left(b_{n}-x\right) & \text { if } x \notin \mathfrak{C}, \text { and } x \in I_{n}=\left(a_{n}, b_{n}\right) \text { for some } n, \\ 0 & \text { if } x \in \mathfrak{C},\end{cases}
$$

where $k_{a_{n}, b_{n}}$ is a positive constant depending on $a_{n}$ and $b_{n}$. Next, let $g$ be the function, on $[-1,1]$, given by:

$$
g(x)= \begin{cases}0 & \text { if } x \in \mathfrak{C}, \\ e^{-1 / d(x)} & \text { if } x \notin \mathfrak{C} .\end{cases}
$$

The value of the constant $k_{a_{n}, b_{n}}$ does not affect at all the smoothness of $g$. For instance, in Figures 1 and 2 we used $k_{a_{n}, b_{n}}=1 /\left(b_{n}-a_{n}\right)^{1.8}$. This constant plays the role of a "scaling factor".

We leave as an exercise to the reader to check that $g$ is smooth. Next, we can define our function $f: \mathbb{R} \rightarrow \mathbb{R}$ by extending $g$ in a usual way by making it smooth on $\mathbb{R}$ and by making it not have horizontal asymptotes.

Let us go back to our main construction now. Let $\mathcal{H}$ be a Hamel basis of $\mathbb{R}$ as a $\mathbb{Q}$-vector space such that all elements in $\mathcal{H}$ are positive. Also, let (for $r \in \mathcal{H}$ and $x \in \mathbb{R})$,

$$
f_{r}(x)=e^{r x} \sin (f(x))
$$

Our aim is to show that the algebra generated by the $f_{r}$ 's, $A=\mathcal{A}\left(f_{r}: r \in \mathcal{H}\right)$, is uncountably generated and that every element in $A$ has an uncountable set of zeros. 


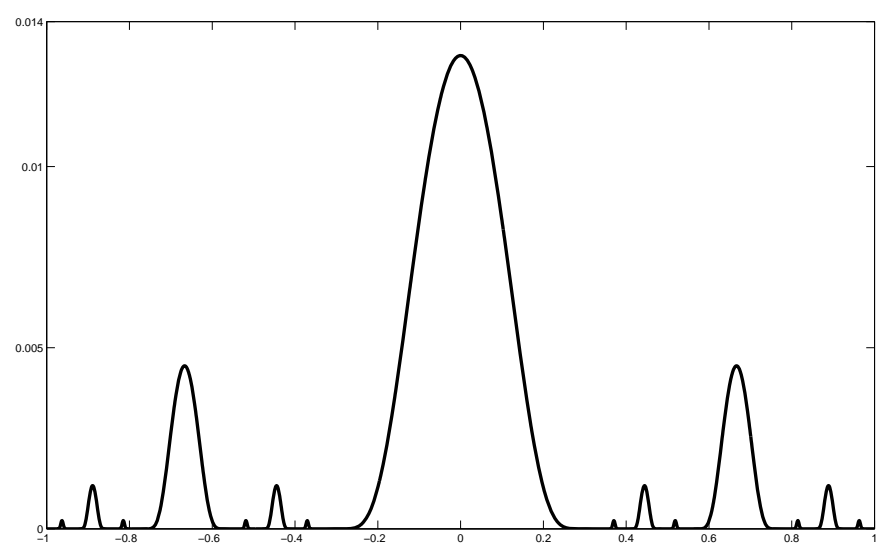

FiguRE 2. Sketch of $g(x)$ on $[-1,1]$.

In order to do so, let $k \in \mathbb{N}, P \in \mathbb{R}\left[z_{1}, z_{2}, \ldots, z_{k}\right]$ be any non-constant polynomial in $k$ real variables, and $r_{1}, r_{2}, \ldots, r_{k} \in \mathcal{H}$. Now we need to see that:

(i.-) $\phi(z):=P\left(f_{r_{1}}, f_{r_{2}}, \ldots, f_{r_{k}}\right)(z)=0$ for every $z \in Z$.

(ii.-) The algebra $A$ is $\mathfrak{c}$-generated.

First, notice that, since $P$ can be written as

$$
P\left(z_{1}, \ldots, z_{k}\right)=\sum_{j=1}^{q} a_{j} \cdot z_{1}^{n_{1, j}} \cdot \ldots \cdot z_{k}^{n_{k, j}},
$$

with $q \in \mathbb{N},\left\{n_{i, j}: 1 \leq i \leq k, 1 \leq j \leq q\right\} \subset \mathbb{N}$, and $a_{j} \in \mathbb{R} \backslash\{0\}$ for every $j \in\{1, \ldots, q\}$, then $\phi$ can be expressed as

$$
\phi(z)=\sum_{j=1}^{q} a_{j} \cdot(\sin f(z))^{\sum_{i=1}^{k} n_{i, j}} \cdot e^{\sum_{i=1}^{k}\left(r_{i} n_{i, j} z\right)}=\sum_{j=1}^{q} a_{j} \cdot(\sin f(z))^{m_{j}} \cdot e^{z s_{j}},
$$

where $m_{j}=\sum_{i=1}^{k} n_{i, j}$ and $s_{j}=\sum_{i=1}^{k} r_{i} n_{i, j}$ for $j \in\{1, \ldots, q\}$.

Once we have that, it is straightforward to check that $\phi(z)=0$ for every $z \in Z$. Next, let us check some properties of the $s_{j}$ 's that appear in the expression of $\phi$. First of all, notice that $s_{j} \neq 0$ for every $j \in\{1, \ldots, q\}$. Indeed, suppose that for some $j \in\{1, \ldots, q\}$ we have $s_{j}=0$. Then, it would be

$$
r_{1} n_{1, j}+r_{2} n_{2, j}+r_{3} n_{3, j}+\cdots+r_{k} n_{k, j}=0,
$$

which contradicts the fact that $\mathcal{H}$ is a Hamel basis. Similarly it can be also shown that $s_{i} \neq s_{j}$ if $i \neq j$. Thus, we can assume without loss of generality, that $s_{1}<$ $s_{2}<\cdots<s_{q}$.

Now, let us show that the set $\left\{f_{r}: r \in \mathcal{H}\right\}$ is algebraically independent. To achieve this, suppose that $\phi \equiv 0$, we shall show that $a_{j}=0$ for every $j \in\{1, \ldots, q\}$. This will amount to $P \equiv 0$, and we will be done. 
If $\phi \equiv 0$, then we would have that

$$
\frac{\phi(z)}{e^{s_{1} z}}=a_{1}(\sin z)^{m_{1}}+\sum_{j=2}^{q} a_{j} \cdot(\sin z)^{m_{j}} \cdot e^{z\left(s_{j}-s_{1}\right)}
$$

is also 0 for every $z \in \mathbb{R}$.

Let, now, take the limit when $z \rightarrow-\infty$. Then, we have that

$$
\begin{aligned}
0 & =\lim _{z \rightarrow-\infty} a_{1}(\sin f(z))^{m_{1}}+\sum_{j=2}^{q} a_{j} \cdot \lim _{z \rightarrow-\infty}(\sin f(z))^{m_{j}} \cdot e^{z\left(s_{j}-s_{1}\right)} \\
& =a_{1} \cdot \lim _{z \rightarrow-\infty}(\sin f(z))^{m_{1}}+\sum_{j=2}^{q} a_{j} \cdot \lim _{z \rightarrow-\infty} \sin (f(z))^{m_{j}} e^{z\left(s_{j}-s_{1}\right)}= \\
& =a_{1} \cdot \sin \left(\lim _{z \rightarrow-\infty} f(z)\right)^{m_{j}}+0,
\end{aligned}
$$

and we obtain that $a_{1}=0$ (since $f$ has no horizontal asymptotes). We can now proceed similarly (dividing now the expression $\sum_{j=2}^{q} a_{j} \cdot(\sin z)^{m_{j}} \cdot e^{s_{j} z}$ by $e^{s_{2} z}$ and taking again limits when $z \rightarrow-\infty)$ and we would obtain that all the $a_{j}$ 's are 0 . Thus, $P \equiv 0$, the set $\left\{f_{r}: r \in \mathcal{H}\right\}$ is algebraically independent, and we are done.

Remark. Notice that this result is the best possible in terms of dimension, since the set of continuous functions has dimension $\mathfrak{c}$. Let us also recall that this construction can also be done using any other types of fractal sets with arbitrary fractal dimension. We chose the Cantor set for convenience.

Acknowledgments. The authors would like to thank the anonymous referee, whose thorough analysis and insightful remarks improved the text. The authors were supported by CNPq Grant 401735/2013-3 (PVE - Linha 2), MTM2012-34341, MEC Project MTM2013-47093-P, and Programa de Investigación y Desarrollo de la UPV, Referencia SP2012070. The third author is also supported by a FPU grant of MEC Project MTM2010-14909.

\section{REFERENCES}

[1] R. M. Aron, D. Pérez-García, and J. B. Seoane-Sepúlveda, Algebrability of the set of nonconvergent Fourier series, Studia Math. 175 (2006), no. 1, 83-90.

[2] R. M. Aron and J. B. Seoane-Sepúlveda, Algebrability of the set of everywhere surjective functions on $\mathbb{C}$, Bull. Belg. Math. Soc. Simon Stevin 14 (2007), no. 1, 25-31.

[3] A. Bartoszewicz and S. Głąb, Strong algebrability of sets of sequences of functions, Proc. Amer. Math. Soc. 141 (2013), 827-835.

[4] L. Bernal-González and M. Ordóñez Cabrera, Lineability criteria, with applications, J. Funct. Anal. 266 (2014), no. 6, 3997-4025, DOI 10.1016/j.jfa.2013.11.014.

[5] L. Bernal-González, D. Pellegrino, and J. B. Seoane-Sepúlveda, Linear subsets of nonlinear sets in topological vector spaces, Bull. Amer. Math. Soc. (N.S.) 51 (2014), no. 1, 71-130, DOI 10.1090/S0273-0979-2013-01421-6.

[6] D. Cariello and J. B. Seoane-Sepúlveda, Basic sequences and spaceability in $\ell_{p}$ spaces, J. Funct. Anal. 266 (2014), no. 6, 3797-3814, DOI 10.1016/j.jfa.2013.12.011.

[7] J. A. Conejero, P. Jiménez-Rodríguez, G. A. Muñoz-Fernández, and J. B. Seoane-Sepúlveda, When the identity theorem "seems" to fail, Amer. Math. Monthly 121 (2014), no. 1, 60-68, DOI 10.4169/amer.math.monthly.121.01.060.

[8] T. Domínguez Benavides, How many zeros does a continuous function have?, Amer. Math. Monthly 93 (1986), no. 6, 464-466, DOI 10.2307/2323473.

[9] P. H. Enflo, V. I. Gurariy, and J. B. Seoane-Sepúlveda, Some results and open questions on spaceability in function spaces, Trans. Amer. Math. Soc. 366 (2014), no. 2, 611-625, DOI 10.1090/S0002-9947-2013-05747-9. 
[10] J. B. Seoane-Sepúlveda, Chaos and lineability of pathological phenomena in analysis, ProQuest LLC, Ann Arbor, MI, 2006. Thesis (Ph.D.)-Kent State University.

Instituto Universitario de Matemática Pura y Aplicada,

Universitat Politècnica de ValènCia,

46022, València, Spain.

E-mail address: aconejero@upv.es

Departamento de Análisis Matemático,

Facultad de Ciencias Matemáticas,

Plaza de Ciencias 3,

Universidad COMPlutense de Madrid,

MADRID, 28040, SPAIN.

E-mail address: gustavo_fernandez@mat.ucm.es

Instituto Universitario de Matemática Pura y Aplicada, Universitat Politècnica de València, 46022, ValÈnCia, Spain.

E-mail address: mamuar1@posgrado.upv.es

Departamento de Análisis Matemático, Facultad de Ciencias Matemáticas,

Plaza de Ciencias 3 ,

Universidad Complutense de Madrid,

MADRID, 28040, SPAIN.

E-mail address: jseoane@mat.ucm.es 